\title{
New geometries for high spatial resolution Hall probes
}

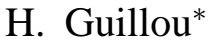 \\ CRTBT-CNRS, laboratoire associ l'universit Joseph Fourier, \\ BP 166, 38042 Grenoble cdex, France \\ A.D. Kent \\ Department of Physics, New York University, \\ 4 Washington Place, New York, New York 10003 \\ G.W. Stupian and M.S. Leung \\ Electronics and Photonics Laboratory, The Aerospace Corporation, \\ P.O. Box 92957, Los Angeles, CA 90009
}

\begin{abstract}
The Hall response function of symmetric and asymmetric planar Hall effect devices is investigated by scanning a magnetized tip above a sensor surface while simultaneously recording the topography and the Hall voltage. Hall sensor geometries are tailored using a Focused Ion Beam, in standard symmetric and new asymmetric geometries. With this technique we are able to reduce a single voltage probe to a narrow constriction 20 times smaller than the other device dimensions. We show that the response function is peaked above the constriction, in agreement with numerical simulations. The results suggest a new way to pattern Hall sensors for enhanced spatial resolution.
\end{abstract}

*Electronic address: herve.guillou@ujf-grenoble.fr 


\section{Introduction}

Hall effect devices are versatile magnetometers operating in a broad range of conditions, such as at high temperature and at high magnetic field. They are routinely used in industry as linear and rotary motion detectors and for power sensing. In the area of fundamental physics Hall magnetometers have recently been used for high sensitivity and spatial resolution magnetic imaging [1], 2], for studies of nanoscale ferromagnetic particles [3] and single molecule magnets [4, 5]. New hybrid devices combining Hall sensors with ferromagnetic materials that behave globally as bipolar switches are also of growing interest [6, 7]. The availability of 2D low electron density and high mobility materials, hence large Hall resistance and signal to noise ratio, make the use of Hall probes based techniques attractive especially when combined with microfabricated devices.

These applications require high spatial resolution, excellent field sensitivity and good magnetic coupling between sample and sensors. One natural way to achieve this is to reduce the dimensions of the sensor active area defined by the intersection of the current and voltage leads. However, as the width of these leads is reduced in size their resistance increases. Higher lead resistance increases device Johnson noise and power dissipation. Hence, up to now, the design of Hall sensors is a compromise between high spatial resolution and good signal to noise ratio. Although numerous studies have been done to optimize the geometry of Hall devices in an homogeneous magnetic field [8], investigation of the Hall effect in inhomogeneous fields has been carried out only in the standard cross shaped geometry [9].

In this paper we propose a new device geometry, based on the careful analysis of the Hall effect, that enhances the sensor spatial resolution without increasing lead resistances. Indeed, we show that high spatial resolution Hall probes can be obtained by reducing the size of one single voltage sensing lead while keeping the other probe dimensions macroscopic. Specifically, we consider the Hall effect in the diffusive transport regime, in which the carrier mean free path is smaller then the smallest sensor dimension. In contrast to the ballistic transport regime, in which for low magnetic fields the Hall response is proportional to the average magnetic field in the sensitive area, in the diffusive regime the Hall response depends strongly on local field inhomogeneities. Numerical simulations have been carried out in our group that show that highly asymmetric Hall probes have sensitive areas localized around the smallest sensing voltage lead [10]. These results have recently been confirmed by other groups [11]. Here we present an experiment that measures directly the Hall response function (HRF) of Hall sensors with symmetric and asymmetric geometries and shows that they have similar spatial resolution. 


\section{Experiment}

The Hall sensors were fabricated in Bi by photolithography. The $2500 \AA$ A thick Bi polycristalline thin films were thermally evaporated on a $200 \AA \mathrm{Ti}$ adhesion layer on sapphire substrates. Photolithography was used to pattern the films into conventional cross like patterns. Although numerous geometries were used, in this article we present only results based on symmetric $2 \mu \mathrm{m} \times 2 \mu \mathrm{m}$ and asymmetric $10 \mu \mathrm{m} \times 5 \mu \mathrm{m}$ current by voltage probes. Bi thin films were characterized at 300 K by measuring the resistivity $R_{\square} \sim 10 \Omega$ and Hall coefficient in a homogeneous magnetic field $R_{H} \sim 0.5 \Omega / \mathrm{T}$. Because of fluctuating deposition conditions and impurity rates, these values vary a little from sample to sample. The mean free path, evaluated using the drude model is about $\lambda_{f} \sim 0.1 \mu \mathrm{m}$ setting the lower bound for probe dimensions in order to be in the diffusive regime. The Hall effect was shown to be linear in homogeneous magnetic fields until 2.5 T. The sign of the Hall coefficient $R_{H}=V_{H} / I$ changes between room temperature and liquid nitrogen temperature. The strong dependence of the Hall coefficient on temperature is due to the non-trivial transport mechanism in Bi that involve several pockets of electrons and holes on the Fermi surface with temperature dependant mobilities. For an isotropic material and two types of carriers, the Hall coefficient is : $R_{H}=\left(p \mu_{p}^{2}-n \mu_{n}^{2}\right) /\left(e \times\left(p \mu_{p}+n \mu_{n}\right)^{2}\right)$ and can change sign [8]. Thus the change of sign in the Hall coefficient of $\mathrm{Bi}$ probes is not surprising and indeed has been noticed earlier by several authors [12, 13, 14, 15, 16].

All the local magnetic measurements presented in this work were made at room temperature, and no attention was given to the actual sign of the Hall coefficient. The key points for these studies are the value of $R_{H}$ at room temperature and the linearity of the Hall effect for small magnetic fields.

We focus now on probe geometries. Numerical computations [9, 10, 11, 17, 18] show that the Hall response is highly dependent on the field distribution. The HRF ; i.e. the Hall voltage measured as a function of the position of a small $\delta$-function like magnetic field ; was found to be highly peaked above the voltage leads [10, 11]. A design where one voltage lead is reduced to submicron sizes while other dimensions of the probe are kept macroscopic, should thus give a highly peaked HRF on the constricted lead and thus a high spatial resolution sensor. To verify this result experimentally we designed extremely asymmetric probe geometry where : i) current and voltage leads have different dimensions and ii) one voltage lead is reduced to a sub-micron size. To produce such probes we used a Focused Ion Beam to tailor the lead geometry. Indeed with such a technique it is possible to cut the Bi films and substrate at very precise locations with a line width smaller than $0.1 \mu \mathrm{m}$. The FIB was used both to design asymmetric probes by cutting only one voltage lead and symmetric probes by 
cutting all four leads in a "clover leaf" pattern as shown on fig. 1. The leads could have been further constricted but other problems such as robustness against electrical shocks occur. It must also be kept in mind that the transport has to be in a diffusive regime.

After constructing our Hall sensor we place a localized magnetic field with a sub-micron resolution at different positions above the surface of the probe and record the Hall voltage as a function of the field position. To measure the HRF we used the Hall probes as the sample and scan a MFM tip over it while recording both the topography and Hall voltage. The resulting image is directly the HRF convolved with the stray field of the tip which we suppose to be very localized [19, 20]. To remove offsets due to misalignment of voltage probes as well as inhomogeneities in the Bi films, a bridge circuit excited by a alternating current was used to record the Hall response. To avoid pickup a carefully shielded cover was added to the MFM setup and the scanning frequency was much smaller than the excitation current to average several periods of the excitation current for each of the 256 points of one line scan. However some pick-up was always present and a low pass filtering of the image yields workable data as shown on fig. 2 and fig. 3.

\section{Results and discussion}

Fig. 2 shows the Hall response functions of symmetric geometries with an outline of the geometrical shape of the sensor superimposed. The greyscales span the same magnetic field amplitude, namely $\Delta B=2.5 \mathrm{mT}$. The two geometries represented : the cross and "clover leaf" are equivalent in terms of Hall effect in an homogeneous field [8]. For both experiments the measured HRF extends slightly outside the area defined by the intersection of the current and voltage probes. This is in agreement with numerical simulations [10] and analytical studies [20] of the corresponding boundary value problem. The difference in field amplitude between images is due to changes in tip magnetization or tilt with respect to the sensor plane between each image. MFM is known to have a submicron magnetic resolution [19, 20], nevertheless the force on the tip is an average of interactions between the tip and a magnetic sample taking place in a finite volume of space. The suposition that the stray field of the MFM tip is a $\delta$-funtion is also certainly not realistic. Attempts have been made to measure directly the stray field of MFM tips with Hall bars [20], electron holography [19], as well as with other techniques. The deconvolution procedure is neither simple nor unique. However, in a first approximation, the magnetized tip is approximated by a magnetic dipole tilted from the $z$-axis. In fig. Za a, on the right side of the probe, the return field of the magnetic dipole is observed as darker areas extending about $2 \mu \mathrm{m}$ away from probe's edges. Such return field has never been observed 
and it shows that the stray field of MFM tips extends over rather large areas. HRF cross sections of symmetric geometries perpendicular and parallel to the excitation current are shown on fig. Ta and fig. 5]a respectively. The full width at half maximum is about twice the probes characteristic size. It is slightly larger in the perpendicular cross section (fig. \#a). The actual measured sensitive area is thus twice as big as the surface defined by the intersection of voltage and current probes. It is simply understood by the presence of a nonzero current density in the voltage probes on a characteristic length of the probes width. The current provides sufficient charge carriers to create a local Hall potential. It is worthwhile to notice in fig. 5a the negative magnetic field corresponding to the above mentioned dark areas.

The HRF of asymmetric geometries are shown in fig. 3. The greyscales have also been adjusted to span the same magnetic field amplitude: $\Delta B=1.6 \mathrm{mT}$. For the initial asymmetric $10 \times 5 \mu \mathrm{m}^{2}$ geometry (fig. 3a), although highly inhomogeneous the HRF is peaked above the voltage probes. Inhomogeneities within the Bi films lead to inhomogeneous current distributions and therefore inhomogeneous HRF. The tilted dipole character of the magnetized tip also leads to an asymmetry in Hall response between the top and bottom voltage probes. The Hall voltage is the convolution of the magnetic field distribution and the actual HRF, for the bottom lead the return field of the tip is crossing the Hall sensor in the middle of the sensitive area whereas for the top lead it is crossing the sensor in a less sensitive area yielding a different Hall voltage. For the constricted geometries (fig. 3 $\mathrm{b}$ and fig. B $\mathrm{c}$ ) the HRF is definitely more peaked above the constriction. The position of the FIB cut is important since the amplitude of the HRF is different in the two images. This is attributed again to a difference in the current density distributions. The FIB cut made in the $10 \mu \mathrm{m} \times 0.25 \mu \mathrm{m}$ (fig. $3 \mathrm{c}$ ) is further away from the current lead than the cut made in the $10 \mu \mathrm{m} \times 0.5 \mu \mathrm{m}$ (fig. $3 \mathrm{~b}$ ) yielding a smaller current density at the constriction and thus a smaller Hall response. This indicates that the Hall response is very sensitive to the local current density. It is illustrated in fig. $5 \mathrm{~b}$ where it is peaked above the corners of the $5 \mu \mathrm{m}$ voltage probe i.e. zones of high current densities.

Fig. $4 \mathrm{~b}$ and fig. $5 \mathrm{~b}$ illustrate that the reduction of one single voltage probe greatly enhances the HRF just above the constriction and therefore the spatial resolution of the sensor, defined as the full width half maximum of the response function. The measured resolutions for the asymmetric probes are respectively for the $5 \mu \mathrm{m}, 0.5 \mu \mathrm{m}$, and the $0.25 \mu \mathrm{m}$ constrictions about $4 \mu \mathrm{m} \times 10 \mu, 2 \mu \mathrm{m} \times 4 \mu \mathrm{m}$ and $2 \mu \mathrm{m} \times 2 \mu \mathrm{m}$. In the case of the $0.5 \mu \mathrm{m}$ FIB cut, the high sensitivity area is almost the same as the one measured for the symmetric $0.5 \mu \mathrm{m} \times 0.5 \mu \mathrm{m}$ cross. The exact shape of the peak in the measured response is intimately connected to the film inhomogeneities, the magnetic field profile, the rounded boundaries and to the non-linearities [11]. It is thus difficult to model quantitatively. We therefore 
focus on qualitative observations to explain our results.

The increasing use of small scale Hall devices follow the need for high spatial resolution imaging

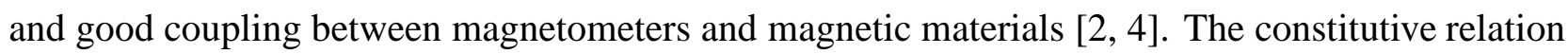
relating local current $J$, electric field $E$ and external magnetic field $B$ in the diffusive regime is:

$$
J=\sigma E+\mu_{H}(J \times B)
$$

where $\sigma$ is the conductivity, $\mu_{H}$ the Hall mobility. In 2D it was shown, that the electric potential in the probe must be a solution of the equation :

$$
\nabla^{2} \phi=-\mu_{H} \nabla B_{z} \cdot(\nabla \phi \times \vec{z})
$$

with the following boundary conditions : i) at conducting boundaries, the tangential component of the electric field is zero, i.e., $\partial \phi / \partial t=0$; and ii) at insulating boundaries, the normal component of the current is zero, i.e., $\partial \phi / \partial n-\mu_{H} B_{z} \partial \phi / \partial t=0$. If $B_{z}$ is uniform, the equation for the electric potential becomes the Poisson equation and the solution is uniquely determined by the boundary conditions, i.e, the fields at the boundaries. In the opposite case there is a source term in the equation. That source term is the key point of our analysis. Indeed in the limit of small magnetic fields, it acts as a distribution of electric dipoles perpendicular to the local electric field. The dipole character of the source term was already noticed in the 1960s [17]. In the case of homogeneous magnetic fields the dipole density is constant throughout the sensor and leads to macroscopic opposite surface charge densities at the boundaries. On the contrary, for a field distribution smaller than the sensor characteristic dimensions, the source dipole distribution is localized within the probe where there is a current density and a varying magnetic field. The enhancement of the HRF in the neighborhood of the constricted lead has two main origins. First, the source term changes rapidly when crossing the constriction perpendicularly to the zero field current distribution. The constriction is a good weak link : there is almost no current flowing through it and the reference equipotential is in its vicinity. Second, the dipolar character of the source term in equation 2 is mostly effective nearby the sensing probe since it is short ranged in nature. These facts, verified numerically [9, 10, 11] and now experimentally show that the geometry of planar Hall device can be optimized to provide a high spatial resolution by scaling down a single probe.

In conclusion, we have measured the HRF function of planar Hall sensors of different geometries. Specifically, we studied an asymmetric design where one single sensing probe is reduced to a narrow constriction. We showed that this design yields identical spatial resolution as the standard reduced cross pattern in agreement with numerical simulation. It validates also the dipole interpretation of 
the Hall effect in inhomogeneous magnetic fields. Although FIB is not the only way to achieve highly asymmetric geometries, it provides a versatile technique to pattern a sensor. It should reveal its full power in fundamental studies of magnetic nanoparticles, for example, to cut sensing probes near the position of particles on a macroscopic sensor.

\section{Acknowledgements}

The research at NYU was supported by the NSF Grant No. DMR-0103290 and a NSF US-France Cooperative Research Grant DMR-9729339. 


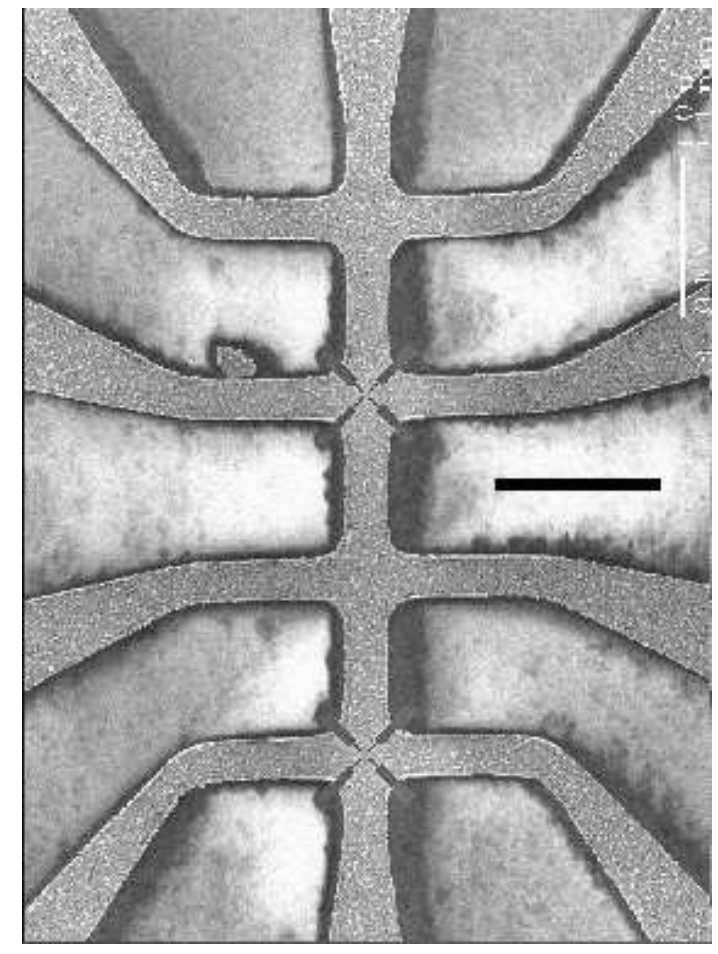

(a)

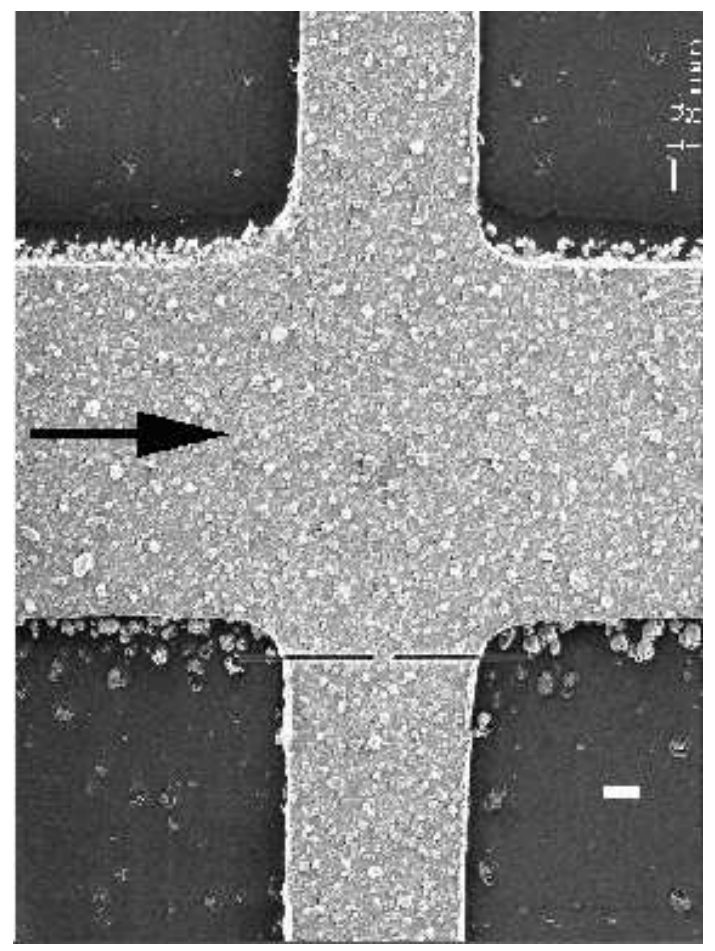

(b)

Figure 1: SEM micrographs of standard cross shaped Hall probes whose geometry has been tailored with FIB.

a) nominal $2 \mu \mathrm{m} \times 2 \mu \mathrm{m}$ geometry symmetrically reduced to $0.5 \mu \mathrm{m} \times 0.5 \mu \mathrm{m}$ (bottom) and $0.25 \mu \mathrm{m} \times 0.25 \mu \mathrm{m}$ (third cross). Black bar in figure is $10 \mu \mathrm{m}$ in length. b) nominal $10 \mu \mathrm{m} \times 5 \mu \mathrm{m}$ geometry asymmetrically reduced to $10 \mu \mathrm{m} \times 0.5 \mu \mathrm{m}$. The black arrow shows the direction of the excitation current. White bar $=1 \mu \mathrm{m}$.

[1] Chong B. K., Zhou H., Mills G., Donaldson L., and Weaver J.M.R., J. Vac. Sci. Technol. A 19(4), 1769 (2001).

[2] Oral A., Bending J., and Henini M., Appl. Phys. Lett. 69(9), 1324 (1996).

[3] Kent A.D., von Molnar S., Gider S., and Awschalom DD, J. Appl. Phys. 76(10), 6656 (1994).

[4] Bokacheva L., Kent A.D., and Walters M.A., Phys. Rev. Lett. 85(22), 4803 (2000).

[5] del Barco E., Kent A.D., Rumberger E.M., Hendrickson D.N., and Christou G., preprint Europhys. Lett. (2002), cond-mat/0209167.

[6] Johnson M., Bennett B.R., Yang M.J., Miller M.M., and Shanabrook B.V., Appl. Phys. Lett. 71(7), 974 (1997).

[7] Monzon F.G., Johnson M., and Roukes M.L., Appl. Phys. Lett. 71(21), 3087 (1997). 


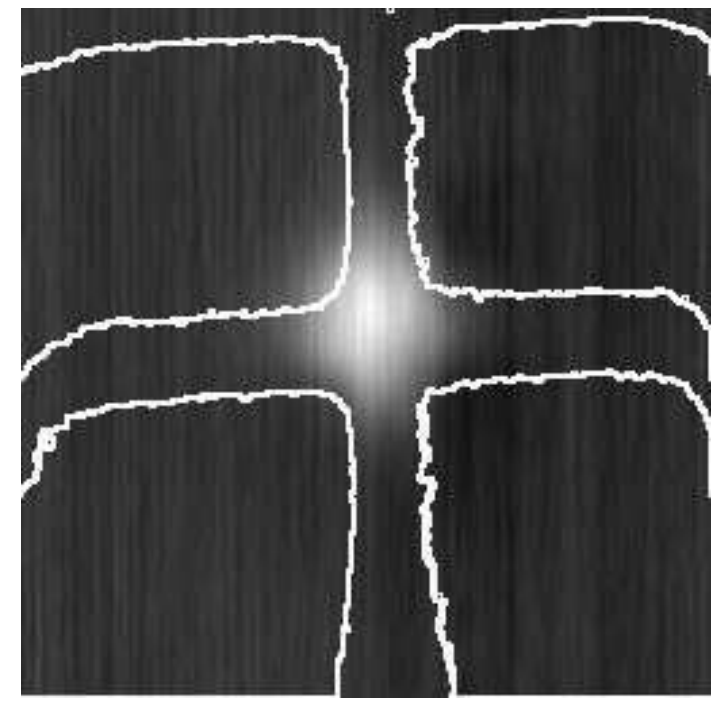

(a)

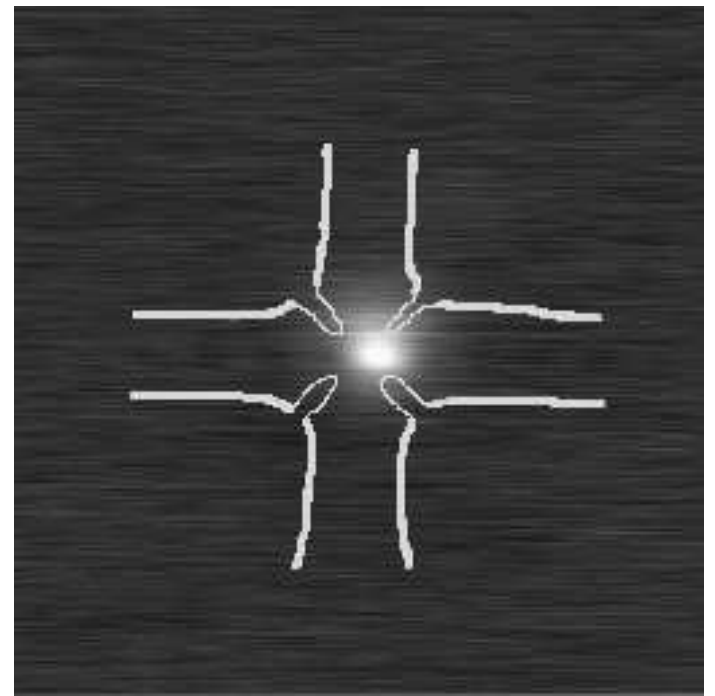

(b)

Figure 2: Hall response of symmetrical probes. The scan range is $19 \mu \mathrm{m}$ and the greyscale spans $2.5 \mathrm{mT}$ in both images. a) sensitive area is $2 \mu \mathrm{m} \times 2 \mu \mathrm{m}$; b) sensitive area is $0.5 \mu \mathrm{m} \times 0.5 \mu \mathrm{m}$. The white lines superimposed on the Hall response image outline the edges of the topographic image. The minor shift between the topographic and the Hall response images stems from several sources. The tilt of the magnetized tip is one of them.

[8] Popovic R.S., Hall effect devices (Adam Hilger, 1991).

[9] Bending S.J. and Oral A., J. Appl. Phys 81(8), 3721 (1997).

[10] Liu S., Guillou H., Kent A.D., Stupian G.W., and Leung M.S., J. Appl. Phys. 83(11), 6161 (1998).

[11] Cornelissens Y.G. and Peeters F.M., The response function of a Hall magnetosensor (2001), preprint cond-mat/0112302.

[12] Boffoué M.O., Lenoir B., Jacquot A., Scherrer H., Dauscher A., and Stölzer M., J. Phys. Chem. of Solids 61, 1979 (2000).

[13] Butenko A.V., Sandomirsky V., Schlesinger Y., Shvarts Dm., and Sokol V.A., J. Appl. Phys. 83(3), 1266 (1997).

[14] Buxo J., Saleh M., Sarrabayrousse G., Dorville G., Berty J., and Brieu M., Revue Phys. Appl. 15, 961 (1980).

[15] Kochowski S. and Opilski A., Thin Solid Films 48, 345 (1978).

[16] Inoue M., Tamaki Y., and Yazi H., J. Appl. Phys. 45, 1562 (1974).

[17] Hlasnik I. and Kokavec J., Solid-State Electronics 9, 585 (1966).

[18] Ibrahim I.S., Schweigert V.A., and Peeters F.M., Phys. Rev. B 57, 15416 (1998). 


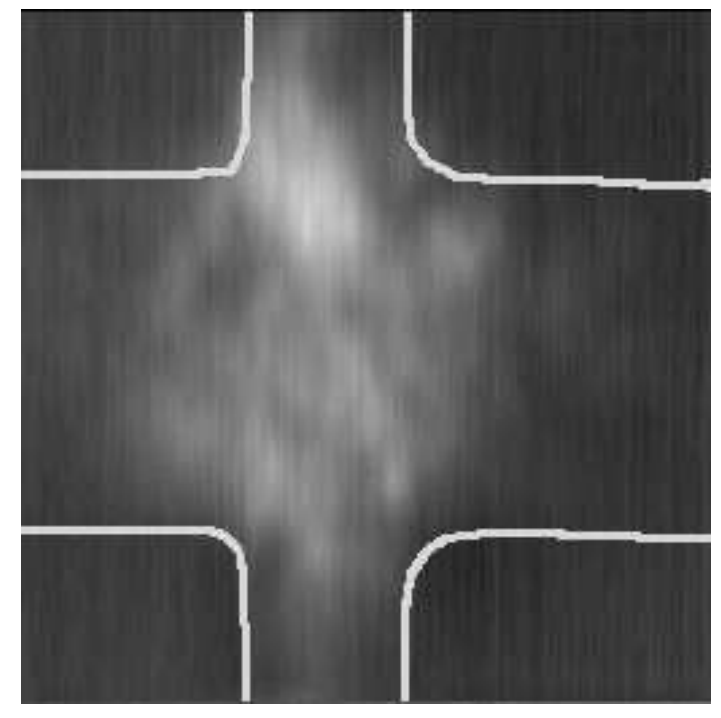

(a)

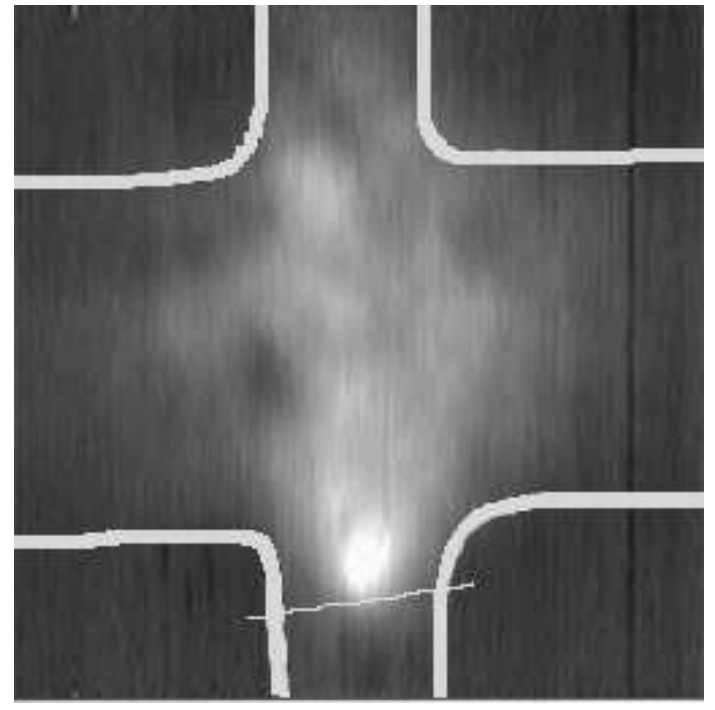

(b)

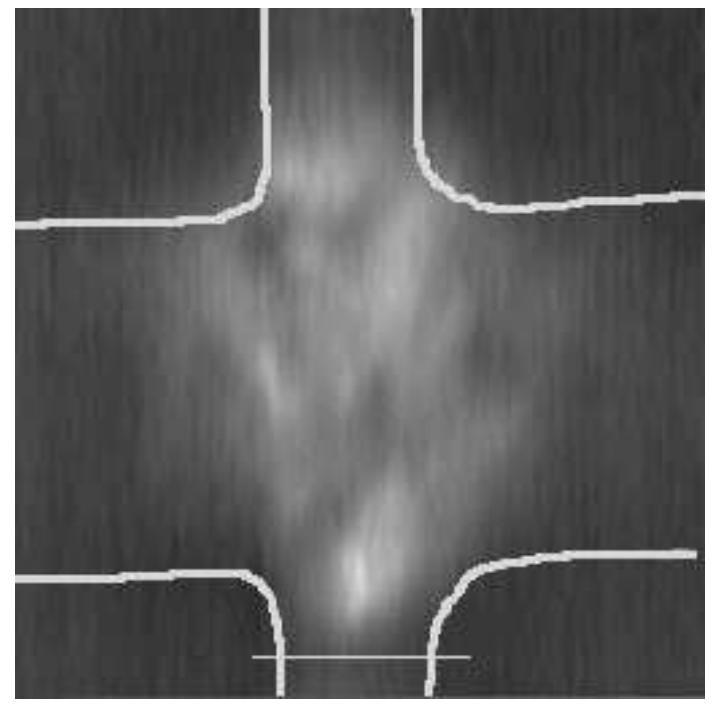

(c)

Figure 3: Hall response of assymetrical probe geometries. The scan range is $19 \mu \mathrm{m}$ and the greyscales span $1.6 \mathrm{mT}$. Lithographic dimensions $10 \mu \mathrm{m} \times 5 \mu \mathrm{m}$ probe a); asymmetrical FIB constricted $10 \mu \mathrm{m} \times 0.5 \mu \mathrm{m}$ probe b) and $10 \mu \mathrm{m} \times 0.25 \mu \mathrm{m}$ probe $\mathrm{c}$ ). The thin white lines indicate the position of the FIB cut.

[19] Streblechenko D. G., Scheinfein M.R., Mankos M., and Babcock K., IEEE Trans. Mag. 32(5), 7954 (1996).

[20] Thiaville A., Belliard L., Majer D., Zeldov E., and Miltat J., J. Appl. Phys. 82(7), 3182 (1997). 


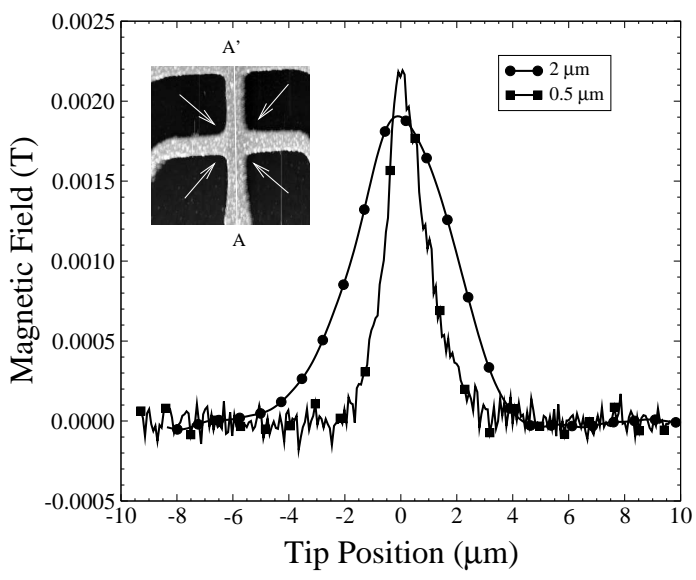

(a)

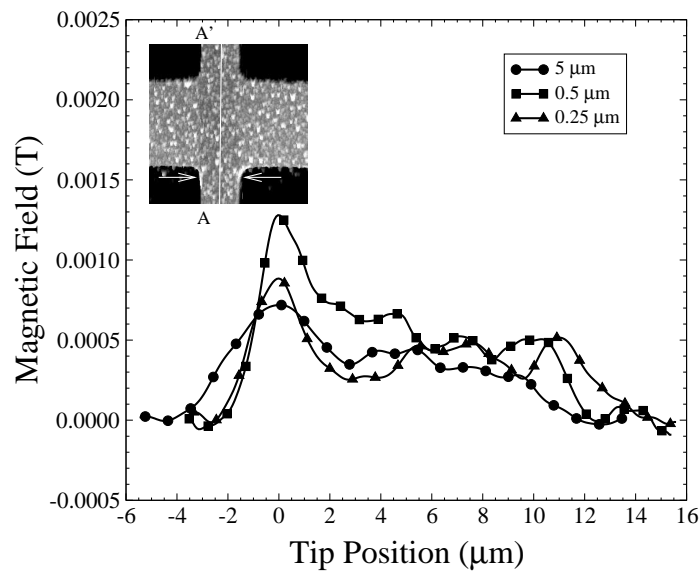

(b)

Figure 4: Cross section of the Hall response function perpendicular to the mean current direction in the case of : a) a symmetrical probe, and b) an asymmetrical probe. The Hall response is measured along the white line $A A^{\prime}$. The white arrows indicate the position of FIB cuts.

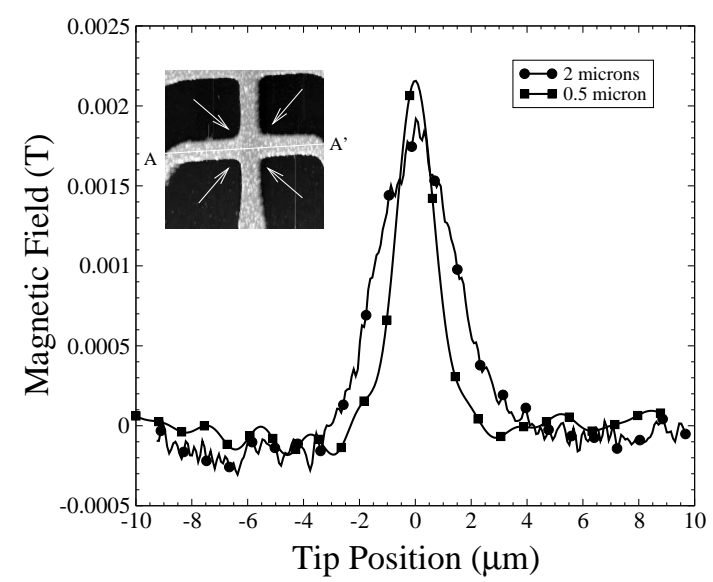

(a)

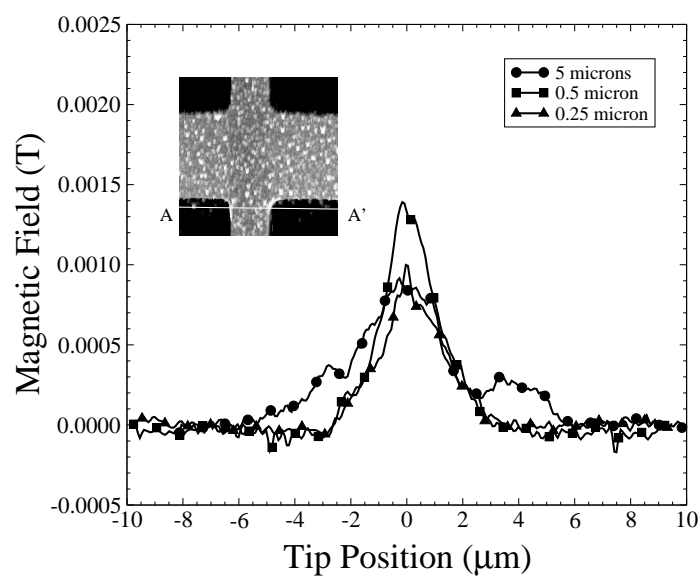

(b)

Figure 5: Cross section of the Hall response function parallel to the average current direction. In case of a) a symmetrical probe, and b) an asymmetrical probe. 